\title{
Yoga therapy and breast cancer
}

Yoga is being utilized with positive results in breast cancer patients as an intervention for lymphedema. This treatment has some medical and psychological benefits to offer. The majority of the studies have been undertaken only after extreme challenges facing breast cancer patients have already been identified. Several studies are bordered on the benefits that Yoga treatment offers to a breast cancer patient. This article is undertaken to bridge that gap on the effectiveness of the Yoga treatment for lymphedema in breast cancer patients. Such effectiveness can be assessed through the evaluation of a number of individuals with breast cancer who have availed the use of Yoga for the treatment of lymphedema. This process calls for surveying and interviewing of breast cancer patients whom Yoga has helped.

Lymphedema is a condition that manifests when the lymphatic system is compromised due to localized fluid and tissue swelling brought about after the disruption triggered by the lymphatic system. Edema, pain, and on several occasions swelling, all characterize signs and symptoms of Lymphedema. Over 140 million individuals around the world experience lymphedema following breast cancer intervention. There are many causes that can make one susceptible to acquire lymphedema which are classified into primary and secondary causes. In primary cause, an individual acquires the ailment through genetic since it can be inherited. In secondary cause, one acquires the disease through injury of the lymphatic system.

\section{KEYWORDS: Iymphedema, breast cancer, yoga, physical therapy, intervention}

\section{Introduction}

Yoga has been used as an intervention for lymphedema in patients who have breast cancer. Research indicates that yoga therapy has positive effects on the treatment process. The benefits are both psychological and medical. Most studies are conducted retrospectively, after assessing the severe challenges that cancer patients have experience. Some studies investigate the benefits of using yoga as an intervention for patients. Similarly, research has been conducted to examine the importance of using yoga therapy in the treatment of lymphedema. Nevertheless, no studies have considered the efficacy and effectiveness of this treatment. The purpose of the present review paper is to fill the gap in the literature by assessing how effective yoga therapy has been to reduce lymphedema in breast cancer patients.

\section{Literature review}

Several studies have recognized the positive impact of a gentle yoga program on those who are coping with cancer. There are medical and psychological benefits of therapeutic yoga. The medical benefits to patients include improved sleep, stronger immune system, better pain management, standardized endocrine function, increased musculoskeletal strength, decreased blood pressure, enhanced balance, and relaxed nervous system. The psychological benefits include: self-acceptance, reduced anxiety and depression, reduced aggression, improved impulse control, and healthy coping approaches. Consequently, many hospitals now include yoga and meditation as part of their integrative medicine programming. Research also addresses decreased function and the impact of self-image that have not been described before. Furthermore, research illustrates the multidimensional conceptualizations of healing, the implications of self-care, and the blurring of boundaries between methods and interventions. This review paper moves beyond the body as it is understood mechanistically and beyond the body of scholarly work on approaches and interventions.

Researchers advocated for the use of yoga therapy in breast cancer patients. In previous studies
James M McKivigan*

Physical Therapist and Chiropractor Touro University, Henderson, Nevada *Author for correspondence:

James.McKivigan@tun.touro.edu 
conducted in 2007, the authors came up with an integrative medicine program used to treat lower limb lymphedema by employing the use of yoga [1]. Their 2014 study, which examines yoga protocol in the course of managing breast cancer, the authors draw the conclusion that yoga plays the same role in central manual lymph drainage as Foldi's method. Doctors used yoga and breathing as an intervention, one which patients may adopt individually in treating breast cancer-related lymphedema. They found that yoga therapy helps patients manage their pain; yoga exercises help in chest expansion and in improving the range of movement (2014) [1].

Other doctors carried out research on yoga management of breast cancer that is related to lymphedema. They used a randomized controlled pilot trial and found a positive correlation between the practice of yoga and managing BCRL [2]. Secondary arm lymphedema has been affecting women after they have been cured of breast cancer, making them in need of a lifelong therapy. The authors estimate that no less than $20 \%$ of women fall into this category. The study concluded that yoga intervention is helpful as it works to decrease tissue induration of the upper arm that is affected. This technique was found to be effective when compared to the control group [2].

Beyond the Body is an insight from an Iyengar yoga program for women with disabilities resulting from breast cancer, conducted by Thomas et al. (2014). The program was developed for women with lymphedema purposed to help them understand arm mobility, become aware of posture, and combat fatigue. The participants reported positive benefits from yoga, which had advanced their understanding of the loss associated with disability. They further stated that yoga enhanced their experience of self. This study addresses the long-term impact of lymphedema on women's emotional and physical health especially regarding arm disability, and topic that has been unnoticed in the literature [3]. To date, researchers have focused on lymphedema while ignoring the effects of pain and motion restrictions. These latter two conditions can be quite debilitating, and although they may be more prevalent than lymphedema or comorbid with lymphedema, their effects are largely undocumented. Pain, motion restrictions, and lymphedema may all affect women's work, their leisure activities, and their relationships with their families [3].
Wanchai's et al. (2010) study is part of a larger research program documenting the interdisciplinary research group that is focused on the development of person-centered creative practices or interventions to address the gaps in cancer survivorship research. Yoga enhances self-image and assists the individual in dealing with posture, loss of gross motor function, and fatigue; this process, in turn, helps in the improvement of arm morbidity. The sensation becomes linked to a more sophisticated conceptualization of well-being; they now feel healthy in their arms. Some patients reported that yoga improves flexibility and helps them cope mentally with their disability. Patients also report increased function after the yoga treatment program [4]

Galantino et al. studied the effects of yoga on functional results (FR) in breast cancer survivors. The study investigated whether yoga was effective in the course of managing pain and other symptoms that breast cancer patient's experience. The authors used a single-arm pilot to evaluate data. Based on the FR measurement, the participants for this study recorded an improvement in balance; flexibility, as measured by FR; and pain management [5].

Research conducted by Neil Piller identified that the lymphatic system has a difference in tissue pressure. This difference is imperative as yoga improves lymphedema flow. The elderly may be more susceptible to lymphedema because they have limited respiratory and skeletal muscle movement capacities. Piller's study indicates that patients with breast cancer are more vulnerable to lymphedema [6].

Wellness coaching is considered an important component in treating breast cancer survivors. Galantino et al. endorse wellness coaching and traditional group support to improve the overall quality of life for survivors. Their preliminary results reveal a significant improvement in the depression subscale used. The authors recommend that survivors should engage in healthy behaviors. Yoga therapy is one of the healthy behaviors as its effectiveness in helping patients has been supported by research.

Galantino et al. (2012) further compares wellness coaching with traditional social support. They assert that wellness coaching is more important than traditional social support mechanisms in treating survivors, as the former has a greater positive impact on quality of life. The author interviewed twenty participants; 
the patients' responses were positive for the study [5]. Galantino et al. assert that wellness coaches have played a significant role in health promotion. Moreover, wellness coaching can be used to empower employees who may be prone to severe diseases to live healthier lifestyles. Similarly, yoga therapy can be employed in the place of traditional health support to empower breast cancer patients so that they might lead healthier lifestyles [5].

Although there are high quality wellness programs in Canada, multidisciplinary treatment programs are not widely available. The chronic nature of disability after breast cancer means that patients rely heavily on selfcare. They are at a disadvantage because they cannot access multidisciplinary services. The result is that they have to endure suffering from the disease which can only be maintained by the patient taking care of their needs. With this contextual knowledge in mind, Galantino et al. (2012) began working with women experiencing disability after breast cancer with a view of developing interventions that are directly informed by participants. The ultimate objective was to create interventions that are accessible and can be undertaken independently without care providers. Yoga has been shown to have positive impact to many illnesses but has rarely been explored when it comes to treating women with disability after breast cancer. He also determined that yoga is well suited to addressing the complexity of disability after breast cancer because it is both a physical and a leisure activity, giving it the potential to become personally meaningful [5].

\section{Discussion}

Lymphedema is a disease caused by a compromised lymphatic system. The condition occurs due to localized fluid retention and the swelling of tissue after injury to the lymphatic systems. The symptoms are characterized by edema, pain, and swelling. Statistics show that the condition affects nearly 140 million people around the world. Numerous causes and prevalence conditions may render one a potential target for this condition. Its causes are categorized into two: primary and secondary. The main reason is when a person inherits the disease, as lymphedema is hereditary. The secondary reason is when the lymphatic system is injured.

Piller (2013) determined that approximately of 1.3 people out of 1000 people around the world are affected with lymphedema. This figure adds up to nearly 140 million people globally. Thus the condition is an issue of concern given a large number of individuals that are affected. Research into lymphedema is necessary to protect these patients from numerous challenges that they face as a result of the condition. Pillar notes that likelihood of developing lymphedema of the arm after having breast cancer ranges from three to sixty per cent. The risk of developing lymphedema of the legs after cancer in the groin or lower abdominal section is approximately 30 percent. Thus it is clear that lymphedema is highly associated with cancer and breast cancer in particular. Piller's study is imperative to this research as it addresses concerns over the extent to which the condition affects people who are breast cancer patients [6]. Narahari et al. (2014) support the hypothesis that yoga therapy is functional as an intervention for patients who have breast cancer. However, his study fails to relate the breast cancer with the effectiveness of using Yoga. Their research is essential as the study included experiments that have used yoga therapy in treating lymphedema conditions, such as lower limb lymphedema [1].

Loudon et al. (2014) used a randomized controlled pilot trial and recorded positive results. The authors found that women who have been affected by breast cancer require lifelong therapy. Loudon's findings are crucial to this review paper as they propose the use of yoga therapy as the lifelong treatment that these patients need. Various benefits can be deduced from Loudon et al., and thus it is important to integrate the controlled pilot trial adopted by Loudon et al. to measure the effectiveness of yoga therapy in pain management for breast cancer patients. Loudon et al. further demonstrates that the randomized controlled pilot trial is more efficient than using a control group. There is no need to use a control group for the present review paper [2].

From the studies presented, it is evident that yoga therapy is a critical intervention strategy in treating lymphedema in breast cancer patients. The effectiveness of yoga intervention can be assessed by evaluating the number of people that have been helped by this method. Moreover, it will be imperative to interview and examine patients to ascertain whether yoga has reduced their lymphedema. There are limitations to these studies. One of the limitations is the difficulty of finding participants to contribute to the research. Another limitation which hinders investigation is financial constraint. 


\section{Conclusion}

In conclusion, the study of the efficacy of yoga therapy on lymphedema in breast cancer patients is beneficial not only to the field of lymphedema intervention, but also to the wider society. Yoga has been used as an intervention for lymphedema in patients who breast cancer. Some studies advocate for the use of yoga therapy in treating breast cancer patients who are also affected by lymphedema. However, no study has examined how effective yoga therapy is in treating patients that are victims of both lymphedema and breast cancer. This study will be beneficial as it will positively support the use of yoga therapy in treaty lymphedema among breast cancer patients. 


\section{References}

Narahari SR, Aggithaya MG, Thernoe L, Bose KS, Ryan TJ. Yoga protocol for treatment of breast cancer-related lymphedema. Int. J. Yoga, 9(2), 145155 (2016).

Loudon A, Barnett T, Piller N, Immink MA, Williams AD. Yoga management of breast cancer-related lymphoedema: a randomised controlled pilot-trial. BMC Complement. Altern. Med. 14(1),
214-127 (2014).

Thomas R, Quinlan E, Kowalski K, Spriggs P, Hamoline R. Beyond the body: insights from an iyengar yoga program for women with disability after breast cancer. Holist. Nurs. Pract. 28(6), 353-361 (2014).

Wanchai A, Armer JM, Stewart BR. Breast cancer survivors' perspectives of care practices in Western and alternative medicine. Oncol. Nurs. Forum. 37(4),
494-500 (2010).

Galantino ML, Desai K, Greene L, et al. Impact of yoga on functional outcomes in breast cancer survivors with aromatase inhibitor-associated arthralgias. Integr. Cancer Ther. 11(4), 313-320 (2012).

Piller N. Lymphoedema: causes, prevention and management in older people. Rev. Clin. Gerontol. 23(02), 142-154 (2013). 